\title{
Contribuciones de la Psicología al abordaje de la dimensión humana del cambio Climático en Chile (Primera parte)*
}

\section{Psychology's CONTRIBUtions to ADDRESS THE HUMAN DIMENSIONS OF CLIMATE CHANGE IN CHILE (FIRST PART)}

\author{
Rodolfo Sapiains ArruÉ ${ }^{* *}$ y Ana María Ugarte Caviedes ${ }^{* * *}$
}

\begin{abstract}
*Trabajo financiado por la Comisión Nacional de Investigación Científica y Tecnológica (CONICYT) como parte del Programa de Atracción e Inserción de Capital Humano Avanzado y realizado en el marco del Proyecto Integrando el Conocimiento Psicológico y el Estudio de las Dinámicas Sociales en el Abordaje del Cambio Climático en Chile, también financiado por el Concurso Nacional de Apoyo al Retorno de Investigadores/as desde el Extranjero-Convocatoria 2014

(Folio proyecto: 82140012) y patrocinado por la Facultad de Ciencias Sociales de la Universidad de Chile y por el Centro de Ciencia del Clima y la Resiliencia (CR) 2.

${ }^{* *}$ Doctor en Factores Psicológicos en la Gestión Ambiental y Magíster en Psicología Comunitaria. Profesor Asistente en el Departamento de Psicología de la Universidad de Chile (Valparaíso, Chile). Miembro del Area Dimensión Humana del

Cambio Climático del Centro de Ciencia del Clima y la Resiliencia (CR) 2. E-Mail: Rodolfo.sapiains@gmail.com

${ }^{* * *}$ Magíster en Ciencias Sociales, mención Sociología y Psicóloga Social-Comunitaria. Profesora en la Escuela de

Psicología de la Facultad de Ciencias Sociales, Jurídicas y Económicas, Universidad Católica Silva Henríquez. Santiago, Chile. E-Mail: amuc@u.uchile.cl

Facultad de Ciencias Sociales de la Universidad de Chile. Santiago, Chile.
\end{abstract}

\section{RESUMEN}

Múltiples estudios señalan que para abordar integralmente la problemática del cambio climático es fundamental incorporar factores psicológicos y sociales en el diseño, implementación y evaluación de estrategias de mitigación y adaptación. Estos factores resultan claves para incrementar la importancia del cambio climático en la agenda pública, favorecer un mayor involucramiento ciudadano y fortalecer la resiliencia individual, social e institucional, así como el impacto de las políticas. No obstante, en Chile el estudio de los aspectos psicológicos del cambio climático es muy limitado.

Por su extensión este trabajo se presenta en dos partes. En esta primera parte se presenta una revisión bibliográfica que explora los principales ámbitos del cambio climático en los que la Psicología puede contribuir para comprender las complejidades del problema. Esto se organiza en cuatro grandes ejes: (1) la comunicación del cambio climático, (2) el estudio de creencias, actitudes, valores y conductas relacionadas con el problema, (3) la identificación de facilitadores y barreras psicológicas para la implementación de prácticas de mitigación y adaptación y (4) los impactos del cambio climático en la salud mental. Estos ejes constituyen un marco de referencia para el posterior desarrollo de ámbitos de acción que se apliquen específicamente al contexto chileno.

Palabras clave: Cambio climático; Psicología, Chile; Conductas pro-ambientales; Adaptación.

\section{ABSTRACT}

Multiple studies show that the integration of social and psychological factors in the design, implementation and evaluation of mitigation and 
adaptation strategies is paramount to address the complexities of climate change. Such factors influence both the perception of the problem and the responses to it in every social system. Considering a multi-level approach encompassing institutional, communitarian and personal dimensions, the inclusion of these psychological aspects can help to increase the importance of climate change in society, favor a deeper community engagement, improving public policies, and strengthen individual, social and institutional resilience. Although Chile is one of the most vulnerable countries in the world regarding climate change impacts, presenting seven out of nine vulnerability criteria, the study of the social and psychological dimensions of climate change in our country is limited and its importance for policy-making generally unknown. In such a context we organized a literature review aimed at exploring how Psychology can contribute to address the human dimensions of climate change in Chile considering a wide range of topics. To do this a reasonable high number of specialized databases and scientific journals were used. As a result of this work we expect to encourage scholars from diverse disciplines, not only psychologist, to dig deeper on these issues in the Chilean and Latin American context to fill this gap in the knowledge. The literature review showed that most research has been conducted in developed countries such as United States, Australia, and United Kingdom. This is expected considering the high levels of political polarization about climate change in those countries, either regarding the causes of the problem, the actions needed to address it, or even if it is happening or not. Contrariwise, we only found a reduced number of articles addressing specifically the psychological aspects of climate change in both Latin America and Chile. Interestingly, mainly scholars from other disciplines generally related to environmental management, planning, Sociology and other social sciences, rather than psychologists, have conducted those studies in the region, most of them focused on issues such as social vulnerability, inequalities, poverty alleviation, political participation and agriculture in rural and indigenous communities in the context of droughts or water management related issues. Results of this review are presented in two parts covering five main topics reflecting the most relevant issues identified in the interaction between psychological factors and climate change in the literature. Part one presents a short introduction on climate change impacts in Chile and the high level of vulnerability of our country given by geographic, environmental and social factors. Then we explain the link between Psychology and environmental problems in general, describing the main issues covered by two specialized sub disciplines called Environmental Psychology and Conservation Psychology. After that we present four sections summarizing the main findings of the literature review. These include the complexities of climate change communication; beliefs, values, attitudes and behaviors; psychological drivers and barriers for climate change action; and the emergent study of the impacts of climate change and mental health. This sets the basis for a framework to organize and encourage research in this area and to integrate this knowledge in policy-making in the Chilean context. Overall, this review suggests that psychology's contributions to address climate change challenges will improve our understanding of the complex relationship between people and nature in general and society and climate change in particular. However, to have a significant impact in policy-making, psychological studies need to be part of wider multidisciplinary teams and work at multiple levels, specially close to communities and public institutions avoiding an excessive experimentalism and academicism preventing isolation from society.

Key words: Climate change; Psychology; Chile; Pro-environmental behaviors; Adaptation.

\section{INTRODUCCIÓN}

De acuerdo al Ministerio del Medio Ambiente, Chile cumple con siete de los nueve criterios establecidos para ser considerado un país vulnerable a los efectos del cambio climático: tiene áreas de borde costero de baja altura, zonas áridas y semiáridas, zonas con cobertura forestal y zonas expuestas al deterioro forestal. Es un país propenso a desastres naturales, también a la sequía y la desertificación. Presenta zonas urbanas con contaminación atmosférica y zonas de ecosistemas 
frágiles, incluidos los sistemas montañosos (Oficina de Cambio Climático, 2014). Las proyecciones consideran un aumento de temperatura en todo el territorio nacional, con un gradiente de mayor a menor, de norte a sur y de la Cordillera de los Andes al Océano Pacífico. Se espera que el mayor calentamiento se verifique en la zona norte grande y en altura, sobre la Cordillera de los Andes. Para el período cercano, entre los años 2011 y 2030, se proyectan disminuciones de precipitación entre $5 \%$ y $15 \%$, para las latitudes $27^{\circ} \mathrm{S}$ a $45^{\circ} \mathrm{S}$, esto es, entre la cuenca del Río Copiapó y la cuenca del Río Aysén. También se prevé un aumento en la probabilidad de eventos de sequía e intensificación de eventos intensos de lluvias, especialmente a partir de la segunda mitad del Siglo XXI. Es muy probable que la duración, frecuencia y / o la intensidad de los períodos cálidos o las olas de calor aumenten en casi todas las zonas continentales (Magrin et al., 2014; Oficina de Cambio Climático, 2014).

Para enfrentar estos escenarios a nivel global y local el IPCC (2014) ha enfatizado que el abordaje del cambio climático implica no solo la reducción de gases de efecto invernadero a nivel de las grandes industrias y países contaminantes, sino que también requiere cambios significativos en múltiples aspectos de la vida cotidiana, como el uso de energía, el manejo del agua, o la producción y el consumo de alimentos. En este contexto, la implementación de adecuadas estrategias de adaptación y mitigación excede las ciencias biofísicas y demanda cambios políticos, institucionales, sociales, culturales y finalmente conductuales, ante los cuales las ciencias sociales y la Psicología pueden jugar un papel fundamental; particularmente esta última, dada su alto potencial en ámbitos relacionados con intervenciones grupales e individuales orientadas al cambio de actitudes y conductas (Landini, 2012).

En Chile, desde los años 90 diferentes gobiernos han desarrollado planes nacionales y sectoriales para enfrentar los nuevos escenarios climáticos. Sin embargo, la consideración e integración de la dimensión humana del problema ha sido muy limitada. Asuntos cla- ves como la influencia de los sistemas de valores y creencias en la percepción y respuestas al cambio climático, la desigual distribución de vulnerabilidades y exposición a condiciones climáticas cambiantes o extremas, impactos en la salud mental, la integración del conocimiento local y científico, las posibilidades reales de participación en sistemas de gobierno ambiental, entre muchos otros aspectos no han ocupado un lugar importante en la política del cambio climático o no han sido abordados en profundidad.

Por otra parte, más allá de que en Chile exista un consenso con respecto a las causas del cambio climático, su importancia y la magnitud de los impactos proyectados (Ministerio del Medio Ambiente, 2015), las temáticas ambientales en general y el cambio climático en particular, siguen siendo percibidos como temas secundarios con respecto a otros problemas sociales (CEP, 2015). Por ejemplo, en la zona centro-sur, particularmente en la región del Bío Bío se percibe que el cambio climático tiene un innegable carácter antrópico y se asocia con una mayor frecuencia de eventos climáticos catastróficos. No obstante, y a pesar de una alta percepción de vulnerabilidad, este tema ocupa una posición solo intermedia entre las preocupaciones ambientales de la población (Infante, A.L. \& Infante, F.C., 2013; Rojas, 2012; Rojas \& Parra, 2010).

En este contexto, el estudio de la dimensión humana del cambio climático y en particular de los aspectos psicológicos es muy relevante tanto para incrementar la importancia del problema en la agenda pública, como para favorecer un mayor involucramiento de la ciudadanía y finalmente fortalecer tanto la resiliencia individual, social e institucional como el impacto de las políticas de cambio climático.

Considerando que en Chile la investigación científica en esta área es muy limitada, el objetivo de este estudio fue explorar los principales ámbitos del cambio climático en los que la Psicología puede contribuir para comprender las complejidades del problema en el contexto chileno y de ese modo favorecer el desarrollo de estrategias, políticas y 
planes más efectivos. Con este fin, se presenta una revisión bibliográfica sobre investigaciones relacionadas con la Psicología y cambio climático y se revisan estudios de caso desarrollados en Chile y en Latinoamérica que relevan los aspectos sociales del problema.

Para la búsqueda se utilizaron las siguientes bases de datos: APA Psyc Net, Scopus, Proquest, Web of Science, Jstor, EBSCO, Wiley, Scielo y Springer. Para la revisión en Chile y Latinoamérica se utilizaron como palabras clave: Climate change and Chile and Psychology*; Global warming and Chile and Psychology*; Climatic change and Chile; Cambio climático y Chile; Calentamiento Global y Chile; Climate change and Latinamerica and psycholo*; Global warming and latinamerica and psycholo*; Climate change and latinamerica and psycholo*, Cambio climático y Latinoamérica y Psicología; Cambio climático y Latinoamérica.

Los resultados fueron organizados en cinco secciones, las cuales se desarrollan luego de una breve contextualización de la relación entre Psicología y problemas ambientales. Se comienza con las complejidades de la comunicación del cambio climático, luego creencias, valores, actitudes y conductas frente al cambio climático, facilitadores y barreras psicológicas, cambio climático y salud mental, y se identifican los ámbitos de acción más relevantes o propios del contexto latinoamericano. Posteriormente se revisan los actuales planes de acción ante el cambio climático en Chile y estudios de caso realizados en el país, identificando los aspectos sociales y psicológicos en cada uno de ellos. Finalmente se proponen las líneas de investigación y áreas temáticas desde las cuales la Psicología puede contribuir a enfrentar las complejidades del cambio climático en el contexto nacional.

\section{LA RELACIÓN ENTRE LA PSICOLOGía Y LOS PRO- BLEMAS AMBIENTALES}

Para muchos autores la sobrepoblación, el sobreconsumo y el uso de tecnologías con- taminantes constituyen las principales causas de los problemas ambientales en el mundo moderno. Se trata de fenómenos socio-ambientales que tensionan la relación entre el ser humano y la naturaleza y que reflejan complejidades culturales, valóricas e ideológicas que se expresan en decisiones y conductas que pueden dañar significativamente el medio ambiente y a otros seres humanos (Ehrlich, P.R. \& Ehrlich, A.H., 2008; Gaston, 2005; Oskamp, 2000; Palumbi, 2001; Penn, 2003). Problemas como la contaminación del aire, del agua o de la tierra, las amenazas para la biodiversidad y el cambio climático reflejan las consecuencias de una compleja, interdependiente y generalmente poco sustentable relación entre las personas y su medio ambiente.

La Psicología Ambiental, disciplina especializada en el estudio de estas temáticas, ha abordado estas problemáticas en al menos dos sentidos: el estudio de los impactos del ambiente natural (aunque también físico y socio-cultural) sobre las personas (e.g., salud mental, productividad, aprendizaje, relaciones sociales) y el estudio de los impactos de las personas sobre el medio ambiente (Bell, Greene, Fisher \& Baum, 2001; Cassidy, 1997; Gifford, 1987). El interés por promover una relación más sustentable con la naturaleza se ha traducido en una creciente productividad académica en esta área (Vleck \& Steg, 2007) y en el desarrollo de una subdisciplina aún más especializada, conocida como Psicología de la Conservación (Conservation Psychology) cuyos principales objetivos son entender por qué las personas se comportan de tal forma que ayudan o dañan el medio ambiente y cómo promover una conducta más responsable con la naturaleza (Clayton \& Myers, 2009; Saunders, 2003).

El estudio de los aspectos psicológicos del cambio climático que se presenta a continuación puede situarse en el marco de la Psicología Ambiental o la Psicología de la Conservación (Conservation Psychology) aunque también puede ser en otras áreas como la Psicología Social, Educacional o en estudios sobre salud mental, comunicación y sobre la percepción del riesgo. 
LAS COMPLEJIDADES DE LA COMUNICACIÓN DEL CAMBIO CLIMÁTICO

El creciente interés por los factores psicológicos asociados al cambio climático se puede explicar por la continua polarización existente en países desarrollados con respecto a las causas del fenómeno (i.e., proceso natural versus producido por actividades humanas), si está ocurriendo o no, o sobre la magnitud de las consecuencias esperadas (e.g., Leiserowitz et al., 2014; Leviston, Price, Malkin \& McCrea, 2014; McCright, Dunlap $\&$ Marquart-Pyatt, 2015). Esto a su vez se ha traducido en serias dificultades para alcanzar acuerdos globales y dentro de cada país con respecto a cómo enfrentar el problema.

Una de las preguntas fundamentales abordada por la Psicología es por qué el consenso dentro de la comunidad científica (Cook et al., 2013) con respecto al cambio climático no se ha traducido en acuerdos dentro de ciertos países desarrollados (e.g., Estados Unidos, Australia) a pesar de los esfuerzos por comunicar la evidencia científica sobre las causas principalmente antropogénicas y la necesidad de desplegar respuestas efectivas y en el corto plazo.

En este contexto se han desarrollado numerosas investigaciones acerca de los factores que explican cómo las personas perciben el cambio climático y cómo responden a éste. Uno de los resultados más importantes es la evidencia de que la información científica es necesaria pero no suficiente para incrementar el apoyo a las políticas sobre el cambio climático. El modelo de déficit según el cual las personas no cambian su actitud o conducta hacia un problema, básicamente se debe a que no tienen suficiente información o no entienden la que existe, esto ha guiado la comunicación del cambio climático desde la comunidad científica (Bain, Hornsey, Bongiorno \& Jeffries, 2012). Los intentos por comunicar ideas y / o problemas complejos utilizando un lenguaje menos técnico y más cercano a la gente no han generado los resultados esperados. El modelo de cognición cultural (cultural cognition) (e.g., Kahan, Jenkins-Smith \& Bramanc, 2011; Norgaard, 2006; Whitmarsh,
2011) ha demostrado cómo las personas filtran información que resulta conflictiva con su sistema de creencias y valores. Esto implica que en temáticas controvertidas o con respecto a respuestas que demanden cambios significativos en los estilos de vida, la calidad de la información tiene un valor secundario o nulo si esta representa una amenaza para la forma de relacionarse las personas con el mundo (Hart \& Nisbet, 2012). En el caso del cambio climático, las acciones generalmente demandadas desde la comunidad científica tienden a poner en entredicho aspectos centrales de las sociedades modernas como el libre mercado y el consumismo, lo cual explicaría las resistencias para aceptar no solo las causas del problema, sino también su existencia en los grupos que más defienden el modelo económico dominante. Esto es aún más relevante considerando que algunos estudios sugieren que tratar de convencer a quienes mantienen una posición opuesta sobre un problema entregándoles más evidencia científica puede incluso incrementar el rechazo hacia el problema y la polarización, aun entre quienes tienen altos niveles de educación (e.g., Corner, Whitmarsh \& Xenias, 2012; Hart \& Nisbet, 2012; Kahan et al., 2012).

Para enfrentar estas dificultades la literatura revisada sugiere al menos tres ámbitos de acción. Por una parte, la segmentación de audiencia que se basa en la identificación de distintos grupos dentro de la sociedad a partir del análisis de factores psicológicos y sociales asociados a distintas posiciones frente al cambio climático, lo cual es utilizado no solo para entender las diferencias, sino también para desarrollar estrategias adaptadas a dichos grupos o considerando elementos transversales a la sociedad (e.g, Hine, Reser, Morrison et al., 2014; Hine, Reser, Phillips et al., 2013; Maibach, Leiserowitz, Roser-Renouf \& Mertz, 2011; Sherley, Morrison, Duncan \& Parton, 2014).

Otro ámbito de acción y relacionado con lo anterior son los estudios sobre encuadre (framing) en los que se trata de activar aquellos elementos considerados como más relevantes con el fin de motivar cambios actitu- 
dinales o conductuales que favorezcan respuestas más efectivas al cambio climático (e.g., Bain et al., 2012; Gifford \& Comeau, 2011; Howell, Capstick \& Whitmarsh, 2016; Sapiains, Beeton \& Walker, 2016). Finalmente estudios cualitativos en la línea de las narrativas (e.g., Daniels \& Endfield, 2009; Drury, Homewood \& Randall, 2010; Moser, 2010) y de las representaciones sociales del cambio climático (e.g., Alvear-Nárvaez, Ceballos-Sarria \& Urbano-Bolaños, 2015; Correa, 2015; González Gaudiano, 2012; Meira-Cartea, 2013; Meira-Cartea \& Arto-Blanco, 2014) han sido propuestos para entender las complejidades psicológicas y sociales del problema, el compo- nente subjetivo de la percepción del riesgo y las múltiples barreras psicológicas que limitan el impacto del saber científico en la sociedad.

\section{CREENCIAS, VALORES, ACTITUdES Y CONDUCTAS FRENTE AL CAMBIO CLIMÁTICO}

Los estudios revisados muestran que tanto en la percepción del cambio climático como en las respuestas que se implementan (y en las que no se practican), los sistemas de creencias y valores juegan un rol fundamental. Esto se asocia con modelos ampliamente utilizados en la Psicología para entender cómo las conductas pro-ambientales se desarrollan (o no) y responder a preguntas fundamentales como la inconsistencia entre preocupación por el medio ambiente y estilos de vida no sustentables. Muchos de estos modelos están basados en la teoría de la acción planificada desarrollada por Ajzen (1991). Esta teoría establece que las conductas son el resultado principalmente de la intención de ejecutar una acción, la cual está determinada por tres factores: actitudes (evaluación positiva o negativa que cada persona hace con respecto a la conducta en cuestión), normas subjetivas (adecuación de la conducta dentro de la sociedad y la presión social o expectativas para ejecutarla) y la percepción de control (posibilidades percibidas de ejecutar dicha conducta en función de experiencias previas y posibles barreras). El modelo supone que altos niveles en estas tres variables incrementan la posibilidad de ejecutar dicha conducta. Por ejemplo, una actitud favorable hacia el ahorro de agua, en un contexto donde dicha práctica se valora y donde la persona siente que lo puede hacer, incrementa las posibilidades de que la acción se implemente. A su vez estas tres variables son influenciadas por creencias con respecto a cada una de ellas.

Otro modelo ampliamente utilizado es el desarrollado por Schwartz (1977), el cual propone que las normas sociales altruistas se traducen en conductas dependiendo de dos variables: (1) la conciencia con respecto a las consecuencias de la conducta y (2) la percepción de responsabilidad sobre el bienestar de los otros. En el contexto ambiental esto implica que, por ejemplo cuando las personas perciben que su producción personal de basura es excesiva y está afectando la calidad de vida de otras personas y al mismo tiempo sienten que es su responsabilidad hacer algo para que esas personas vivan en mejores condiciones, su conducta podría ser modificada.

Un tercer ejemplo es la teoría desarrollada por Stern, Dietz, Abel, Guagnano y Kalof (1999) quienes trabajaron sobre las ideas de Schwartz y Ajzen. En este modelo se plantea la importancia inicial de diferentes tipos de valores (i.e., biocéntricos, altruistas y egoístas), diferentes tipos de creencias con respecto al $\mathrm{NEP}^{1}$, las consecuencias adversas sobre objetos o personas valoradas, la creencia con respecto a la capacidad de hacer algo y finalmente, un sentido de obligación, todo lo cual explicaría el desarrollo de cuatro tipos de conductas pro-ambientales (i.e., activismo

1 El NEP (New Ecological Paradigm) se refiere a la idea de que el surgimiento del movimiento ambientalista está asociado a la aceptación creciente de una visión del mundo más ecológica en la cual las personas tienen una mayor conciencia de las consecuencias de sus acciones sobre la naturaleza. Para mayores antecedentes sobre el concepto y la forma en que se mide se puede revisar Dunlap y Van Liere (1978). 
ambiental, ciudadanía ambiental, apoyo a políticas ambientales y conductas pro-ambientales en la vida privada).

Estas teorías han sido utilizadas ampliamente para abordar problemas claves de la dimensión humana con respecto al cambio climático: por ejemplo para entender la asimetría entre las intenciones de las acciones proambientales y su bajo impacto real (Whitmarsh, 2009), para explorar el rol de valores no ambientales (i.e., altruistas) en la adopción de estilos de vida más sustentables (Howell, 2013), o en el estudio de factores que incrementan la disposición para apoyar estrategias para el cambio climático en general (Dietz, Dan \& Shwom, 2007; Nilsson, von Borgstede \& Biel, 2004). Es decir, se trata de modelos y conceptos que pueden contribuir significativamente a mejorar el entendimiento de la compleja relación que las personas establecen con el problema incluyendo cómo lo perciben y el tipo de respuestas que implementan.

\section{FACILITADORES Y BARRERAS PSICOLÓGICAS}

Diversos estudios han posibilitado la identificación de facilitadores y barreras psicológicas para implementar estrategias de mitigación y adaptación. Por ejemplo, se ha mostrado que el apego al territorio puede tener un impacto positivo en el compromiso con el cambio climático (Devine-Wright, Price \& Leviston, 2015; Scannell \& Gifford, 2013); por el contrario, la comunicación de escenarios catastróficos por sí sola tiende a reforzar una percepción de impotencia sobre el problema y a activar valores egoístas (Aitken, Chapman \& McClure, 2011), por otra parte, en muchos casos las personas simplemente no quieren saber acerca del problema, exhibiendo procesos de negación de la evidencia científica (Norgaard, 2006; Stoll-Kleemann, O'Riordan \& Jaeger, 2001).

Otros estudios han mostrado el impacto negativo de la percepción de incertidumbre sobre el consenso científico o sobre los efectos del cambio climático en la aceptación del problema y / o en la implementación de respuestas (e.g., Corner et al., 2012; Ding, Mai- bach, Zhao, Roser-Renouf \& Leiserowitz, 2011; Whitmarsh, 2011). Se ha mostrado cómo muchos grupos auto-identificados como ambientalistas no presentan patrones de consumo necesariamente más eficientes que otros (e.g, Newton \& Meyer, 2013; Tobler, Visschers \& Siegrist, 2012), y se han estudiado los aspectos políticos y comunitarios que favorecen o desmotivan la acción asociados al fenómeno de los free-riders y la tragedia de los comunes $^{2}$ (Quiemby \& Angelique, 2011).

Gifford (2011) organizó las barreras psicológicas en siete grandes categorías: limitaciones cognitivas, ideologías, comparación con otros, costos hundidos, incredulidad, percepción del riesgo y limitaciones conductuales.

Lorenzoni, Nicholson-Coleb y Whitmarsh (2007) identificaron barreras individuales y sociales, las cuales pueden ser también conscientes o inconscientes. Entre otras, estas incluyen las resistencias para cambiar estilos de vida, la prevalencia de lo económico, la poca valoración de los sacrificios o cambios personales comparados con la responsabilidad de gobiernos e industrias, etc. (ver Cuadro 1).

\section{CAMBIO CLIMÁTICO Y SALUD MENTAL}

Finalmente, existe una creciente investigación sobre los impactos negativos del cambio climático en la salud mental. Berry, Bowen y Kjellstrom (2010) identificaron impactos directos asociados a fenómenos climáticos tales como cambios dramáticos en la tempe-

2 El problema de los free-riders se refiere al aprovechamiento de otros de los sacrificios personales, por ejemplo pagando menos o utilizando más un recurso natural. La tragedia de los comunes propone que cuando las personas actúan individual y racionalmente tienden a poner sus propios intereses por sobre el bien común, lo cual se traduce en un uso abusivo de los recursos naturales. Se puede revisar el trabajo original en Hardin (1968) y una discusión posterior en Ostrom, Burger, Field, Norgaard y Policansky (1999). 
ratura, olas de calor extremo, inundaciones, sequías prolongadas, ciclones. Entre estos impactos se destacan altos niveles de ansiedad, estrés, mayores niveles de agresividad, baja productividad y mayor riesgo de accidentes, todo lo cual se incrementa cuando las personas presentan problemas de salud mental previos. Por otra parte, se identifican efectos indirectos asociados a cambios en el sistema socioeconómico (e.g., baja productividad agrícola), deterioro del entorno natural, problemas de salud física y a los cambios asociados a la implementación de medidas de mitigación (e.g., mayores tiempos de transporte) y adaptación (e.g., trabajar de noche para evitar olas de calor). Doherty y Clayton (2011) identifican también impactos negativos asociados a la exposición de los efectos del cambio climático a través de los medios de comunicación y la inmediatez de las nuevas tecnologías, incluyendo ansiedad, duelo, culpa, desesperanza, negación, o apatía. Al mismo tiempo, también se mencionan impactos sociales y comunitarios, incluyendo mayor violencia por el incremento de las temperaturas; conflictos y problemas entre grupos por competencia sobre recursos naturales (e.g., agua), duelo, ansiedad y sentido de pérdida de identidad asociada a desplazamientos, incremento de tensiones sociales por desigualdades y restricciones de acceso a ecosistemas saludables. Adicionalmente, un estudio conducido por Jones, Wootton, Vaccaro y Menzies (2012) sugiere un impacto negativo del cambio climático en el desarrollo y / o agudización del trastorno obsesivo-compulsivo.

Estos estudios resaltan que estos efectos tendrán mayor impacto en comunidades cuyo sustento está más directamente relacionado con la naturaleza, como en el caso de los agricultores (Morrissey \& Reser, 2007; Polain, Berry \& Hoskin, 2011) y en comunidades in- dígenas que viven en situación de pobreza, con altos niveles de desempleo, alcoholismo, abuso de drogas y otros problemas sociales, especialmente cuando existen traumas y desventajas históricas y para los cuales el deterioro ambiental puede incrementar la sensación de pérdida de identidad o de su patrimonio cultural (Bourque \& Cunsolo Willox, 2014).

Por otra parte, Petrasek, Cunsolo Willox, Forda, Shiwakc y Woodd (2015) han identificado factores protectores tales como poseer un fuerte sentido de pertenencia al territorio, una fuerte conexión con la naturaleza y con la cultura local, la mantención de prácticas tradicionales y tener buenas relaciones con familia y amigos. Al mismo tiempo, el cambio climático puede ofrecer oportunidades para desarrollar capital social, asociatividad y mejor capacidad adaptativa, por ejemplo cuando las comunidades participan activamente en el cuidado de su entorno y en el desarrollo del lugar donde viven, favoreciendo un mejor bienestar emocional y social (Berry, 2009; Berry, Butler et al., 2010).

\section{Conclusiones}

Las cuatro áreas de trabajo desarrolladas en esta revisión presentan un marco de referencia para entender las potenciales contribuciones de la Psicología para el abordaje de la dimensión humana del cambio climático en Chile.

En la segunda parte de este estudio se desarrollará la temática desde el contexto latinoamericano y se presentará una propuesta para la investigación desde esta perspectiva y sus diversas implicancias para el trabajo comunitario y en relación con las políticas públicas para el cambio climático. 
CUADRO 1

BARRERAS PSICOLÓGICAS PARA EL DESARROLLO DE ESTRATEGIAS DE MITIGACIÓN Y ADAPTACIÓN AL CAMBIO CLIMÁTICO

\begin{tabular}{|c|c|}
\hline Categorías & Barreras \\
\hline Cognitivas & $\begin{array}{l}\text { Conflictos ideológicos y / o de valores (e.g., cultural cognition) } \\
\text { Baja percepción de control } \\
\text { Percepción de poca eficacia de las acciones personales } \\
\text { El medio ambiente es importante, pero no una prioridad } \\
\text { Negación o racionalización del problema y disociación (disonancia entre preocupación por el } \\
\text { medio ambiente y estilo de vida no sustentable) } \\
\text { Baja percepción de riesgo } \\
\text { Incertidumbre con respecto al problema (causas y / o soluciones) } \\
\text { Externalización de las responsabilidades } \\
\text { Problema percibido como distante en el futuro o geográficamente lejano } \\
\text { Percepción de falta de tiempo o dinero } \\
\text { Dificultad cognitiva para proyectarse en el futuro y reaccionar a amenazas ambientales comple- } \\
\text { jas }\end{array}$ \\
\hline Relacionales & $\begin{array}{l}\text { Comparación social } \\
\text { Normas sociales } \\
\text { Efecto de los free-riders } \\
\text { Expectativas (sobre la conducta de los otros y la de uno mismo) }\end{array}$ \\
\hline $\begin{array}{l}\text { Afectivas / } \\
\text { Emoocionales }\end{array}$ & $\begin{array}{l}\text { Desinterés o apatía } \\
\text { Insensibilidad ante los problemas ambientales } \\
\text { Fatalismo y / o desesperanza (muy tarde para actuar / nada se puede hacer) } \\
\text { Tipo de vínculo con el lugar donde se vive } \\
\text { Emociones negativas gatilladas por o asociadas al cambio climático (pena, miedo, duelo, enojo, } \\
\text { culpa, frustración) } \\
\text { Presiones de la vida cotidiana que son más concretas e inmediatas que los riesgos del cambio } \\
\text { climático } \\
\text { Desconfianza hacia las fuentes de información (sensacionalismo, exageración) } \\
\text { Desconfianza hacia las instituciones de gobierno y empresas privadas }\end{array}$ \\
\hline Conductuales & $\begin{array}{l}\text { Foco en conductas de bajo costo y bajo impacto (Tokenismo) } \\
\text { Hábitos no sustentables } \\
\text { Acciones pro-ambientales cuyo impacto positivo se minimiza o anula por el aumento de su fre- } \\
\text { cuencia (Efecto rebote) } \\
\text { Resistencia a reducir el uso de ciertos objetos (e.g., auto) debido al alto costo que implicó adqui- } \\
\text { rirlos } \\
\text { Rechazo a políticas y acciones que coarten la libertad individual } \\
\text { Falta o insuficiente feedback sobre el impacto de las acciones personales } \\
\text { Saturación con respecto a las acciones desarrolladas ("estoy haciendo todo lo que puedo hacer") }\end{array}$ \\
\hline
\end{tabular}

Continúa 


\begin{tabular}{|l|l|}
\hline Categorías & \multicolumn{1}{|c|}{ Barreras } \\
\hline Culturales & $\begin{array}{l}\text { Ideologías políticas, creencias religiosas y / o visiones de mundo no sustentables } \\
\text { Tecno-optimismo (sobrevaloración de la tecnología para resolver todos los problemas ambien- } \\
\text { tales) } \\
\text { Promoción y prevalencia de estilos de vida consumistas } \\
\text { Estereotipos negativos asociados a estilos de vida "verdes" }\end{array}$ \\
\hline Externas / & $\begin{array}{l}\text { Falta de acciones políticas efectivas a nivel local, nacional, internacional } \\
\text { Fstructurales }\end{array}$ \\
$\begin{array}{l}\text { Falta de acciones efectivas desde la industria } \\
\text { productos ecológicos, acceso a tecnología, etc.) } \\
\text { Contexto de crisis económica } \\
\text { Falta de liderazgo político (tipos de gobierno) } \\
\text { Ausencia o fiscalización limitada a empresas contaminantes (no son responsabilizadas por sus } \\
\text { acciones -accountability) }\end{array}$ \\
\hline Información & $\begin{array}{l}\text { Falta de información sobre causas y consecuencias } \\
\text { Falta de información sobre impactos locales } \\
\text { Falta de información sobre el tipo de soluciones y sus impactos } \\
\text { Problemas de acceso a la información } \\
\text { Información parcial o contradictoria } \\
\text { Sobrecarga de información } \\
\text { Información en lenguaje técnico y complejo } \\
\text { Acciones basadas en el modelo de déficit de información }\end{array}$ \\
\hline
\end{tabular}

*La información presentada en este cuadro se basa en los trabajos de Gifford (2011), Lorenzoni, Nicholson-Coleb y Whitmarsh (2007) y otros estudios citados. 


\section{REFERENCIAS BIBLIOGRÁFICAS}

Aitken, C., Chapman, R. \& McClure, J. (2011). Climate change, powerlessness and the commons dilemma: Assessing New Zealanders' preparedness to act. Global Environmental Change, 21, 752-760.

Ajzen, I. (1991). The theory of planned behavior. Organizational Behavior and Human Decision Processes, 50, 179-211.

Alvear-Nárvaez, N., Ceballos-Sarria, V. \& UrbanoBolaños, M. (2015). Los jóvenes de la ciudad de Popayán frente al cambio climático. Un estudio desde las representaciones sociales [The young people from Popayán city facing climate change. A social representations study]. Revista Ingenierías Universidad de Medellín, 14(26), 43-56.

Bain, P.G., Hornsey, M.J., Bongiorno, R. \& Jeffries, C. (2012). Promoting pro-environmental action in climate change deniers. Nature Climate Change, 2, 600-603. http://dx.doi.org/10. 1038/nclimate 1532

Bell, P., Greene, T., Fisher, J. \& Baum, A. (2001). Environmental Psychology. Orlando: Hartcourt College.

Berry, H. (2009). Pearl in the oyster: Climate change as a mental health opportunity. Australas Psychiatry, 17(6), 453-456.

Berry, H.L., Bowen, K. \& Kjellstrom, T. (2010). Climate change and mental health: A causal pathways framework. International Journal of Public Health, 55, 123-132. http://dx.doi.org/1 0.1007/s00038-009-0112-0

Berry, H.L., Butler, J.R.A., Burgess, C.P., King, U.G., Tsey, K., Cadet-James, Y.L. \& Raphael, B. (2010). Mind, body, spirit: Co-benefits for mental health from climate change adaptation and caring for country in remote Aboriginal Australian communities. New South Wales Public Health Bulletin, 21(5-6), 139-145.

Bourque, F. \& Cunsolo Willox, A. (2014). Climate change: The next challenge for public mental health? International Review of Psychiatry, 26 (4), 415-422.

Cassidy, T. (1997). Environmental psychology. Behaviour and experience in context. East Sussex: Psychology Press.

CEP. (2015). Estudio nacional de opinión pública [Public opinion national survey], 74. Santiago, Chile: Centro de Estudios Públicos (CEP).

Clayton, S. \& Myers, G. (2009). Conservation psychology: Understanding and promoting human care for nature. Oxford: Wiley-Blackwell.

Cook, J., Nuccitelli, D., Green, S.A., Richardson, M., Winkler, B., Painting, R. \& Skuce, A. (2013). Quantifying the consensus on anthropogenic global warming in the scientific literature. Environmental Research Letters, 8(2), 02 4024. http://dx.doi.org/10.1088/1748-9326/8/2 1024024

Corner, A., Whitmarsh, L. \& Xenias, D. (2012). Uncertainty, scepticism and attitudes towards climate change: Biased assimilation and attitude polarization. Climatic Change 114, 463-478.

Correa, M.I. (2015). Cambio climático y representaciones sociales entre estudiantes de educación superior [Climate change and social representations among terciary education students]. En B. Ortiz \& C. Velasco (Eds.), La percepción social del cambio climático. Estudios y orientaciones para la educación ambiental en México (pp. 107-122). México: Universidad Iberoamericana de Puebla.

Daniels, S. \& Endfield, G.H. (2009). Narratives of climate change: Introduction. Journal of Historical Geography, 35, 215-222.

Devine-Wright, P., Price, J. \& Leviston, Z. (2015). My country or my planet? Exploring the influence of multiple place attachments and ideological beliefs upon climate change attitudes and opinions. Global Environmental Change, 30, 68-79.

Dietz, T., Dan, A. \& Shwom, R. (2007). Support for climate change policy: Social psychological and social structural influences. Rural Sociology, 72(2), 185-214. 
Ding, D., Maibach, E.W., Zhao, X., Roser-Renouf, C. \& Leiserowitz, A. (2011). Support for climate policy and societal action are linked to perceptions about scientific agreement. Nature Climate Change, 1, 462-466.

Doherty, T. \& Clayton, S. (2011). The psychological impacts of global climate change. American Psychologist, 66(4), 265-276. http://dx.doi.org/ 10.1037/a0023141

Drury, R., Homewood, K. \& Randall, S. (2010). Less is more: The potential of qualitative approaches in conservation research. Animal Conservation, 14, 18-24.

Dunlap, R.E. \& Van Liere, K.D. (1978). The new environmental paradigm: A proposed measuring instrument and preliminary results. Journal of Environmental Education, 9, 10-19.

Ehrlich, P.R. \& Ehrlich, A.H. (2008). Nature's economy and the human economy. Environmental and Resource Economics, 39, 9-16. http://dx. doi.org/10.1007/s10640-007-9177-5

Gaston, K.J. (2005). Biodiversity and extinction: Species and people. Progress in Physical Geography, 29(2) (Biodiversity), 239-247.

Gifford, R. (1987). Environmental Psychology. Principles and practices. Newton: Allyn \& Bacon.

Gifford, R. (2011). The dragons of inaction psychological barriers that limit climate change mitigation and adaptation. American Psychologist, 66(4), 290-302.

Gifford, R. \& Comeau, L. (2011). Message framing influences perceived climate change competence, engagement, and behavioral intentions. Global Environmental Change, 21, 1301-1307.

Gonzalez Gaudiano, E.J. (2012). La representación social del cambio climático: Una revisión internacional [Social representation of climate change: An international review]. RMIE, 17 (55), 1035-1062.

Hardin, G. (1968). The tragedy of the commons. Science, New Series, 162(3859), 1243-1248.

Hart, P.S. \& Nisbet, E.C. (2012). Boomerang effects in science communication: How motiva- ted reasoning and identity clues amplify opinion polarization about climate mitigation policies. Communication Research, 39, 701-723.

Hine, D.W., Reser, J.P., Morrison, M., Phillips, W. J., Nunn, P. \& Cooksey, R. (2014). Audience segmentation and climate change communication: Conceptual and methodological considerations. Wiley Interdisciplinary Reviews: Climate Change, 5(4), 441-459. http://dx.doi.org/ $10.1002 /$ wcc. 279

Hine, D.W., Reser, J.P., Phillips, W.J., Cooksey, R., Marks, A.D.G., Nunn, P. \& Glendon, A.I. (2013). Identifying climate change interpretive communities in a large Australian sample. Journal of Environmental Psychology, 36, 229239. http://dx.doi.org/10.1016/j.jenvp.2013.0 8.006

Howell, R.A. (2013). "It's not (just) 'the environment, stupid!" Values, motivations, and routes to engagement of people adopting lower-carbon lifestyles. Global Environmental Change, 23, 281-290.

Howell, R.A., Capstick, S. \& Whitmarsh, L. (2016). Impacts of adaptation and responsibility framings on attitudes towards climate change mitigation. Climatic Change, 136(3), 445-461. http: //dx.doi.org/10.1007/s10584-016-1627-z

Infante, A.L. \& Infante, F.C. (2013). Percepciones y estrategias de los campesinos del secano para mitigar el deterioro ambiental y los efectos del cambio climático en Chile [Dry-land peasant's perceptions and strategies aimed to the mitigation of environmental degradation and the effects of climate change in Chile]. Agroecología, 8(1), 71-78.

IPCC. (2014). Climate Change 2014: Synthesis Report. Contribution of Working Groups I, II and III to the Fifth Assessment Report of the Intergovernmental Panel on Climate Change. En R. K.P. a. L.A.M. Core Writing Team (Eds.), (pp. 151). Geneva, Switzerland: IPCC.

Jones, M., Wootton, B., Vaccaro, L. \& Menzies, R. (2012). The impact of climate change on obsessive compulsive checking concerns. Austra- 
lian \& New Zealand Journal of Psychiatry, 46 (3), 265-270.

Kahan, D.M., Jenkins-Smith, H. \& Bramanc, D. (2011). Cultural cognition of scientific consensus. Journal of Risk Research, 14(2), 147-174.

Kahan, D.M., Peters, E., Wittlin, M., Slovic, P., Larrimore Ouellette, L., Braman, D. \& Mandel, G. (2012). The polarizing impact of science literacy and numeracy on perceived climate change risks. Nature Climate Change, 2, 732735 .

Landini, F. (2012). Expectativas de los agentes de desarrollo rural argentinos sobre la Psicología y sobre la inserción profesional de los psicólogos en el ámbito de la extensión rural [Expectations of Argentinian rural development practitioners on psychology and the professional integration of psychologists in the rural area]. Interdisciplinaria, 29(2), 359-377.

Leiserowitz, A., Maibach, E., Roser-Renouf, C. Feinberg, G., Rosenthal, S. \& Marlon, J. (2014). Climate change in the American mind: Americans' Global warming beliefs and attitudes in November 2013. Yale University and George Mason University New Haven, CT: Yale Project on Climate Change Communication.

Leviston, Z., Price, J., Malkin, S. \& McCrea, R. (2014). Fourth Annual survey of Australian attitudes to climate change: Interim Report. Perth, Australia: CSIRO.

Lorenzoni, I., Nicholson-Coleb, S. \& Whitmarsh, L. (2007). Barriers perceived to engaging with climate change among the UK public and their policy implications. Global Environmental Change, 17, 445-459.

Magrin, G.O., Marengo, J.A., Boulanger, J.P., Buckeridge, M.S., Castellanos, E., Poveda, G., et al. (2014). Central and South America. En V.R. Barros, C.B. Field, D.J. Dokken, M.D. Astrandrea, K.J. Mach, T.E. Bilir et al. (Eds.), Climate change 2014: Impacts, adaptation, and vulnerability. Part B: Regional aspects. Contribution of Working Group II to the Fifth Assessment Report of the Intergovernmental Panel on
Climate Change (pp. 1499-1566). Cambridge, UK and NY: Cambridge University Press.

Maibach, E.W., Leiserowitz, A., Roser-Renouf, C. \& Mertz, C.K. (2011). Identifying like-minded audiences for global warming public engagement campaigns: An audience segmentation analysis and tool development. PLos One, 6(3), e17571. http://dx.doi.org/10.1371/journal.pon e. 0017571

McCright, A.M., Dunlap, R.E. \& Marquart-Pyatt, S.T. (2015). Political ideology and views about climate change in the European Union. Environmental Politics, 25(2), 1-21. http://dx.doi. org/10.1080/09644016.2015.1090371

Meira-Cartea, P. (2013). Representaciones sociales del cambio climático en la sociedad española: Una lectura para comunicadores [Social representations of climate change in spanish society: An interpretation for communicators]. En R. Fernández \& R. Mancinas (Eds.), Medios de comunicación y cambio climático (pp. 59-90). Sevilla: Fénix Editora.

Meira-Cartea, P. \& Arto-Blanco, M. (2014). Representaciones del cambio climático en estudiantes universitarios en España: Aportes para la educación y la comunicación [Representations of climate change in university students in Spain: Contributions to education and communication]. Educar em Revista, Edição Especial, 3, 15-33.

Ministerio del Medio Ambiente. (2015). Primera Encuesta Nacional de Medio Ambiente [The First National Survey on Environmental]. Santiago, Chile: Ministerio del Medio Ambiente.

Morrissey, S. \& Reser, J. (2007). Natural disasters, climate change and mental health considerations for rural Australia. Journal of Rural Health, 15, 120-125.

Moser, S.C. (2010). Communicating climate change: History, challenges, process and future directions. Wiley Interdisciplinary Reviews: Climate Change, 1, 31-53.

Newton, P. \& Meyer, D. (2013). Exploring the attitudes-action gap in household resource con- 
sumption: Does "environmental lifestyle" segmentation align with consumer behaviour? Sustainability, 5(3), 1211-1233.

Nilsson, A., Von Borgstede, C. \& Biel, A. (2004). Willingness to accept climate change strategies: The effect of values and norms. Journal of Environmental Psychology, 24(3), 267-277. http:// dx.doi.org/10.1016/j.jenvp.2004.06.002

Norgaard, K.M. (2006). "We don't really want to know" environmental justice and socially organized denial of global warming in Norway. Organization Environment, 19(3), 347-370.

Oficina de Cambio Climático, Ministerio del Medio Ambiente (2014). Plan Nacional de Adaptación al Cambio Climático [Climate Change Adaptation National Plan] (p. 56). Santiago, Chile: Ministerio del Medio Ambiente.

Oskamp, S. (2000). A sustainable future for humanity? How can Psychology help? American Psychologist, 55(5), 496-508.

Ostrom, E., Burger, J., Field, C., Norgaard, R. \& Policansky, D. (1999). Revisiting the commons: Local lessons, global challenges. Science, 284, 278-282.

Palumbi, S. (2001). Humans as the world's greatest evolutionary force. Science, 293, 1786-1790.

Penn, D.T. (2003). The evolutionary roots of our environmental problems: Toward a Darwinian ecology. The Quarterly Review of Biology, 78 (3) (Evolution and Environment), 275-301.

Petrasek, J., Cunsolo W. A., Forda, J.D., Shiwakc, I. \& Woodd, M. (2015). Protective factors for mental health and well-being in a changing climate: Perspectives from Inuit youth in Nunatsiavut, Labrador. Social Science \& Medicine 141, 133-141.

Polain, J., Berry, H. \& Hoskin, J. (2011). Rapid change, climate adversity and the next 'big dry': Older farmers' mental health. Journal Rural Health, 19, 239-243.

Quiemby, C. \& Angelique, A. (2011). Identifying barriers and catalysts to fostering pro-environmental behavior: Opportunities and challenges for community Psychology. American Journal of Community Psychology, 47, 388-396.
Rojas, J. (2012). Impactos sociales y ambientales del cambio climático global en la región del Bio Bío. Desafios para la sostenibilidad del siglo $X X I$ [Social and environmental climate change impacts in the Bio Bio region. Challenges for sustainability of the 21st century]. Concepción: Editorial Universidad de Concepción.

Rojas, J. \& Parra, O. (2010). Cambio climático local: La región del Bío Bío en Chile en contexto global [Local climate change: Bio Bio region in the global context]. Revista Brasileira de Ciências Ambientais, 17, 7-16.

Sapiains, R.P., Beeton, R.J.S. \& Walker, I.A. (2016). Individual responses to climate change: Framing effects on pro-environmental behaviors. Journal of Applied Social Psychology, 46 (8), 483-493.http://dx.doi.org/10.1111/jasp.123 78

Saunders, C. (2003). The emerging field of conservation Psychology. Human Ecology Review, 10(2), 137-149.

Scannell, L. \& Gifford, R. (2013). Personally relevant climate change: The role of place attachment and local versus global message framing in engagement. Environment and Behavior, 45(1), 60-85.

Schwartz, S.H. (1977). Normative influences on altruism. En L. Berkowitz (Ed.), Advances in Experimental Social Psychology (Vol. 10, pp. 221-279). New York: Academic Press.

Sherley, C., Morrison, M., Duncan, R. \& Parton, K. (2014). Using segmentation and prototyping in engaging politically-salient climate-change household segments. Journal of Nonprofit \& Public Sector Marketing, 26(3), 258-280. http: //dx.doi.org/10.1080/10495142.2014.91 8792

Stern, P., Dietz, T., Abel, T., Guagnano, G.A. \& Kalof, L. (1999). A value-belief-norm theory of support for social movements: The case of environmentalism. Human Ecology Review, 6(2), 81-97.

Stoll-Kleemann, S., O'Riordan, T. \& Jaeger, C.C. (2001). The psychology of denial concerning climate mitigation measures: Evidence from 
Swiss focus groups. Global Environmental Change, 11, 107-117.

Tobler, C., Visschers, V.H.M. \& Siegrist, M. (2012). Addressing climate change: Determinants of consumers' willingness to act and to support policy measures. Journal of Environmental Psychology, 32, 197-207.

Vleck, C. \& Steg, L. (2007). Human behavior and environmental sustainability: Problems, driving forces and research topics. Journal of Social Issues, 63(1), 1-19.

Whitmarsh, L. (2009). Behavioural responses to climate change: Asymmetry of intentions and impacts. Journal of Environmental Psychology, 29, 13-23.

Whitmarsh, L. (2011). Scepticism and uncertainty about climate change: Dimensions, determinants and change over time. Global Environmental Change, 21, 690-700.

\section{Facultad de Ciencias Sociales Universidad de Chile Santiago - Chile}

Fecha de recepción: 6 de enero de 2016 Fecha de aceptación: 16 de enero de 2017 
